\title{
National Assessment and Accreditation Council (NAAC)'s accreditation of health sciences institutes: Challenges and the road ahead
}

\author{
Arun Jamkar \\ Former Vice Chancellor of Maharashtra University of Health Sciences and Vice President, i4C. \\ *Corresponding Author: Arun Jamkar \\ Email: jamkar@gmail.com
}

As you are aware, NAAC has come up with a renewed manual to enhance the process of assessment and accreditation for Higher Education Institutions (HEIs) including universities and colleges in the health sciences sector. All the health sciences institutes, which seek accreditation will now have to follow the directives of the new NAAC framework hereafter. I feel, the introduction of the renewed manual which has been composed considering the dynamic demands of the health sciences' sector, will definitely help fuel growth, inspire innovation, incorporate latest technology and research in the curriculum and uphold quality across all health sector universities and colleges.

Among many others, one of the keys benefits of NAAC's renewed manual is that earlier these universities had to use general protocol, however, it was only applicable to university departments and affiliated colleges were not taken into consideration. $\mathrm{We}$ as an Association of Health Professionals universities of India, had approached NAAC to have separate manual editions for universities and colleges to cover their specific requirements.

I am very proud of the fact that the NAAC framework is entirely 'Made in India' and covers requirements that are specific to the Indian health sciences domain and do not blindly follow the western parameters which seek little relevance to our country. The western standards fail to cover the challenges faced by the Indian health sciences sector, and they do not make a provision to offer solutions for these problems.

On the other hand, the renewed manual has been developed based on the feedback of key Indian stakeholders, domain experts, academicians and the strong backdrop of the previous year's manual, hence, it is all-encompassing, and technology driven, thus, catering to the comprehensive requirements of the Indian health sciences domain. Therefore, it is very important for health sciences universities and colleges to understand the new requirements of the manual for NAAC assessment and accreditation.

\section{Here are few challenges and opportunities that the renewed NAAC manual entails \\ Optimizing technology trends}

Universities and colleges should be aware that, NAAC demands well-developed learning ecosystems, as it will ask for clinical skill laboratories and simulator labs. Even Medical Council of India (MCI) had recently asked for this facility in a phased-out manner.

The assessment for accreditation will take place through an ICT enabled new process that is robust, transparent, objective and scalable. Optimizing latest technology will save a lot of time and repetitive efforts. Advanced technology, tools and learning e-resources will help students to get a holistic and enriching learning experience.

\section{Harnessing Innovation}

Apart from having an enriching curriculum with updated syllabus, NAAC insists on innovation in addition to research. Students will also be evaluated on the basis of creativity, analytical skills and processes that would nurture the spirit of innovations in students. NAAC also gives an impetus to innovation incubation centers to encourage out of box thinking and development of breakthrough solutions for critical issues.

\section{Prioritizing student satisfaction}

Under NAAC accreditations, HEIs will offer many valueadded services to students, which they can choose from, based on their interests and inclinations. Besides this, NAAC proforma evaluates and measures the 'student friendly' quotient of every institute.

NAAC insists on mentorship programmes, parent and teacher association and even states about the remedial measures to be taken, wherever necessary. For the very first time, health sciences professional institutes have to submit an online student satisfaction survey, the feedback received will then be utilized to enhance the effectiveness of learning.

\section{Strengthening medical ethics}

I appreciate that NAAC is insisting on implementation of ethics and principles not only in the curriculum but also in research. As the Chair of National Curriculum Implementation committee of UNESCO Bioethics programme of UNESCO Chair of Bioethics (Haifa), I am sure it will enhance the movement of medical ethics education in country and give it the much-required boost. This will lead to ethical value-based education at a national level.

\section{Other critical evaluation parameters}

NAAC also requires evaluation on other important parameters with adequate weightage laid on leadership, vision and mission statement, gender equality, alternate sources of energy, disability friendly environment and others. Hence, health sciences universities and colleges will also have to comply with NAAC's requirements for these critical parameters along with the others mentioned in the framework. 
Given the above-mentioned merits, I think that evaluation of health sciences institutes by NAAC based on the renewed framework for accreditation and assessment will enhance the standard of education offered and open a plethora of opportunities for Indian students. It will definitely act as a catalyst to create better health sciences professionals that will improve the quality of medical care research and offerings, thus, minimizing external dependency and benefiting India. 